\section{Saliva Alternative to Upper Respiratory Swabs for SARS-CoV-2 Diagnosis}

\author{
Rachel L. Byrne, Grant A. Kay, \\ Konstantina Kontogianni, Ghaith Aljayyoussi, \\ Lottie Brown, Andrea M. Collins, Luis E. Cuevas, \\ Daniela M. Ferreira, Tom Fletcher, Alice J. Fraser, \\ Gala Garrod, Helen Hill, Grant L. Hughes, \\ Stefanie Menzies, Elena Mitsi, Sophie I. Owen, \\ Edward I. Patterson, Stacy Todd, \\ Christopher T. Williams, Angela Hyder-Wright, \\ Emily R. Adams, Ana I. Cubas-Atienzar
}

Author affiliations: Liverpool School of Tropical Medicine, Liverpool, UK (R.L. Byrne, G.A. Kay, K. Kontogianni, G. Aljayyoussi, L. Brown, A.M. Collins, L.E. Cuevas, D.M. Ferreira, T. Fletcher, A.J. Fraser, G. Garrod, H. Hill, G.L. Hughes, S. Menzies, E. Mitsi, S.I. Owen, E.I. Patterson, S. Todd, C.T. Williams, A. Hyder-Wright, E.R. Adams, A.I. Cubas-Atienzar); National Institute for Health Research, Leeds, UK (A.M. Collins, A. Hyder-Wright); Liverpool University Hospitals National Health Services Foundation Trust, Liverpool (A.M. Collins, T. Fletcher, A. Hyder-Wright S. Todd)

DOI: https://doi.org/10.3201/eid2611.203283

PCR of upper respiratory specimens is the diagnostic standard for severe acute respiratory syndrome coronavirus 2 infection. However, saliva sampling is an easy alternative to nasal and throat swabbing. We found similar viral loads in saliva samples and in nasal and throat swab samples from 110 patients with coronavirus disease.

$\mathrm{Q}$ uantitative reverse transcription PCR (qRTPCR) is the diagnostic standard for severe acute respiratory syndrome coronavirus 2 (SARSCoV-2), the causative agent of coronavirus disease (COVID-19) (1). Testing usually is conducted on upper respiratory specimens collected using swabs (1-3). However, this method requires multiple samples and has a low sensitivity (4). Swab sampling can cause patients to cough or sneeze, uncomfortable reactions that might also increase transmission risks to healthcare workers (A. Wyllie et al., unpub. data, https://doi.org/10.1101/2020.04.16.2 0067835). Sampling technique proficiency also varies, especially during self-sampling (A. Wyllie et al., unpub. data, https://doi.org/10.1101/2020.0 4.16.20067835), which can result in false negatives. Furthermore, shortages of swabs, transport media, and personal protective equipment limit healthcare capacity to conduct SARS-CoV-2 tests that rely on swab sampling.
Saliva sampling is a noninvasive alternative to upper respiratory swabbing. We compared paired self-collected saliva samples with healthcare worker-collected nasal and throat swab specimens from 110 patients with suspected SARS-CoV-2 infection. This analysis was part of a prospective study (Facilitating a SARS CoV-2 Test for Rapid Triage) at the Royal Liverpool University and Aintree University Hospitals (Liverpool, UK). We recruited participants who had provided written informed consent and had COVID-19 symptoms. The National Health Service Research Ethics Committee (20/SC/0169) approved the study under Integrated Research Application System no. 282147.

Within 24 hours after patient consent, we collected nasal and throat swab specimens containing 1.0 $\mathrm{mL}$ of Amies transport medium (COPAN Diagnostics, https:/ / www.copanusa.com). We also asked participants to funnel their saliva into a sterile cryotube (SARSTEDT, https:/ / www.sarstedt.com). We immediately extracted RNA from the swab samples; we stored saliva samples at $-80^{\circ} \mathrm{C}$ until processing. We extracted viral RNA using the QIAamp Viral RNA Mini Kit (QIAGEN, https:/ / www.qiagen.com) and tested $8 \mu \mathrm{L}$ of extracted RNA using the genesig Real-Time Coronavirus COVID-19 PCR (genesig, https://www.genesig.com). We quantified viral loads using the manufacturer's positive control $\left(1.67 \times 10^{5}\right.$ copies $\left./ \mu \mathrm{L}\right)$ as reference.

Of the 110 adults recruited from April through June 2020, a total of 61 (55.5\%) were women. Most participants were hospitalized; 21 (19.1\%) were discharged to home directly from the emergency department. Overall, 12 (10.9\%) saliva and 14 (12.7\%) nasal and throat swab specimens of 110 paired samples tested positive for

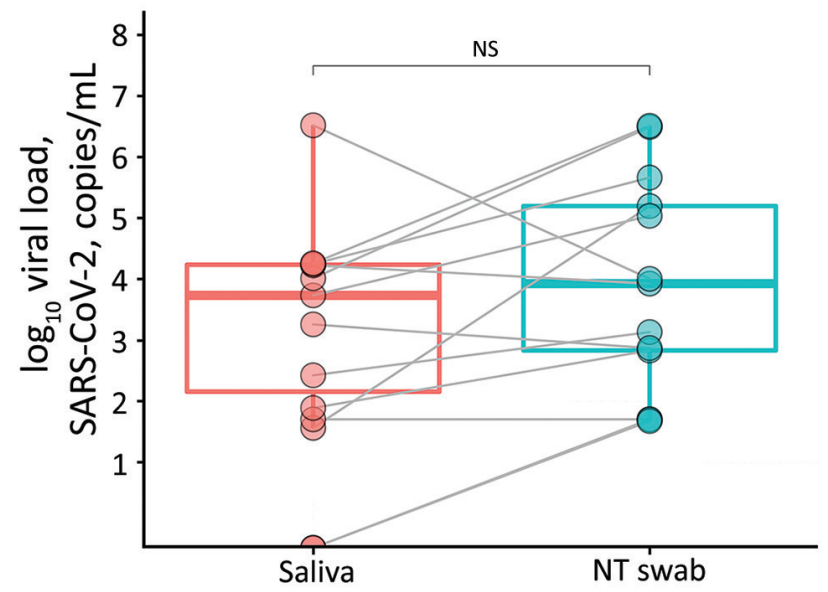

Figure. Viral load (copies/mL) of SARS-CoV-2 RNA recovered from paired saliva samples and nasal and throat swab specimens from 14 patients with coronavirus disease, United Kingdom, 2020. Viral loads are shown on a logarithmic scale. NS, not significant; NT, nasal and throat; SARS-CoV-2, severe acute respiratory syndrome coronavirus 2 . 
SARS-CoV-2 RNA. Viral loads for all samples ranged from 36 to $3.3 \times 10^{6}$ copies $/ \mathrm{mL}$. Overall viral loads were similar among all positive samples (Figure).

Insignificant viral load discrepancies existed among all positive samples ( $p=0.1955$ by Wilcoxon signed-rank paired test). Two patients tested positive $(<10$ copies/mL) on nasal and throat swab samples and negative on saliva samples; the discrepancies might have resulted from the different processing times of the 2 specimens because freeze-thawing can reduce the stability of RNA $(5,6)$.

Saliva sampling can improve SARS-CoV-2 diagnostic techniques. Saliva samples are easier to collect than nasal and throat samples; the technique is noninvasive, presumably preferred by the participant, and does not require sampling proficiency. In addition, saliva sampling does not require swabs and transport media, which have limited availability during the pandemic. Our technique uses a funnel which, although helpful, might not be necessary for sample collection. Our study focused on symptomatic hospitalized participants; further research is needed on saliva sampling for patients with mild and asymptomatic SARS-CoV-2 infection.

Further studies should document the effects of storage and transport on RNA and viral loads. Rapid processing of saliva samples might benefit patients in low- and middle-income countries, where the pandemic is still accelerating and swab availability is limited (7). Furthermore, high-income countries can establish a cold chain for sample transportation. A cold chain could enable home sampling and screening of children who have rejected swabbing. It could also streamline research studies that require repeat sampling.

As rates of SARS-CoV-2 infection increase, we must continue to investigate efficient diagnostic strategies. Easy and effective diagnostic techniques, such as saliva sampling, should be evaluated in certified clinical laboratories.

This article was preprinted at https://www.medrxiv.org/ content/10.1101/2020.07.09.20149534v1.

\section{Acknowledgments}

We are grateful to the participants in the Facilitating A SARS CoV-2 Test for Rapid Triage study for their involvement in this research. We also thank the research nurses from Liverpool University Hospitals National Health Services Foundation Trust and National Institute for Health Research and the Liverpool School of Tropical Medicine team that assisted with the sample collection and processing. We acknowledge the advice, guidance, and community of the Clinical Research Network in the North West Coast.
This study was financially supported by the Department for International Development/Wellcome Trust Epidemic Preparedness coronavirus grant no. 220764/Z/20/Z and Pfizer grant no. WI255862 (D.M.F., E.M., A.C.). E.R.A. and L.E.C. are funded by the National Institute for Health Research Health Protection Research Unit in Emerging and Zoonotic Infections, the Centre of Excellence in Infectious Diseases Research, and the Alder Hey Charity. We also acknowledge the financial support of Liverpool Health Partners and the Liverpool Malawi COVID-19 consortium.

\section{About the Author}

Ms. Byrne is a doctoral candidate at the Liverpool School of Tropical Medicine, Liverpool. Her primary research interest is the application of new molecular diagnostics for the detection of emerging infectious diseases.

\section{References}

1. World Health Organization. Laboratory testing strategy recommendations for COVID-19. 2020 [cited 2020 July 1]. https://www.who.int/publications/i/item/laboratorytesting-strategy-recommendations-for-covid-19-interimguidance

2. Centers for Disease Control and Prevention. Interim guidelines for collecting, handling, and testing clinical specimens from persons for coronavirus disease 2019 (COVID-19). 2020 [cited 2020 Jun 22]. https:/ / www.cdc.gov/ coronavirus/2019-ncov/lab/guidelines-clinicalspecimens.html

3. To KKW, Tsang OTY, Leung WS, Tam AR, Wu TC, Lung DC, et al. Temporal profiles of viral load in posterior oropharyngeal saliva samples and serum antibody responses during infection by SARS-CoV-2: an observational cohort study. Lancet Infect Dis. 2020;20:565-74. https:/ / doi. org/10.1016/S1473-3099(20)30196-1

4. Zhang W, Du RH, Li B, Zheng XS, Yang XL, Hu B, et al. Molecular and serological investigation of 2019-nCoV infected patients: implication of multiple shedding routes. Emerg Microbes Infect. 2020;9:386-9. https:/ / doi.org/ 10.1080/22221751.2020.1729071

5. Ji X, Wang M, Li L, Chen F, Zhang Y, Li Q, et al. The impact of repeated freeze-thaw cycles on the quality of biomolecules in four different tissues. Biopreserv Biobank. 2017;15:475-83. https://doi.org/10.1089/bio.2017.0064

6. Kuang J, Yan X, Genders AJ, Granata C, Bishop DJ. An overview of technical considerations when using quantitative real-time PCR analysis of gene expression in human exercise research. PLoS One. 2018;13:e0196438. https:/ / doi.org/10.1371/journal.pone.0196438

7. Gilbert M, Pullano G, Pinotti F, Valdano E, Poletto C, Boëlle PY, et al. Preparedness and vulnerability of African countries against importations of COVID-19: a modelling study. Lancet. 2020;395:871-7. https://doi.org/10.1016/ S0140-6736(20)30411-6

Address for correspondence: Emily R. Adams, Centre for Drugs and Diagnostics, Liverpool School of Tropical Medicine, Pembroke Place, Liverpool L3 5QA, UK; email: emily.adams@1stmed.ac.uk 